\title{
Reduced expression of SIRT2 in serous ovarian carcinoma promotes cell proliferation through disinhibition of CDK4 expression
}

\author{
YANHUA DU ${ }^{1,2^{*}}, \mathrm{JUN} \mathrm{WU}^{3 *}$, HAIYAN ZHANG ${ }^{1,2},{\text { SHAOBO } \mathrm{LI}^{3} \text { and HONG SUN }}^{1,2}$ \\ ${ }^{1}$ Department of Gynecology, Obstetrics and Gynecology Hospital of Fudan University; \\ ${ }^{2}$ Shanghai Key Laboratory of Female Reproductive Endocrine Related Diseases, Shanghai 200011; \\ ${ }^{3}$ Pathology Center, Shanghai General Hospital/Faculty of Basic Medicine, Shanghai \\ Jiaotong University School of Medicine, Shanghai 200025, P.R. China
}

Received November 24, 2015; Accepted December 1, 2016

DOI: $10.3892 / \mathrm{mmr} .2017 .6183$

\begin{abstract}
The silent information regulator 2 related enzyme 2 (SIRT2) has been reported to have an important role in tumorigenesis. Although two distinct effects of SIRT2 have recently been revealed, which explain opposing expression patterns in different types of cancer, the specific function of SIRT2 in ovarian cancer remains unknown. The present study investigated the expression of SIRT2 in serous ovarian carcinoma (SOC) and its pathogenic mechanism. It was observed that SIRT2 expression in SOC was significantly downregulated when compared with ovarian surface epithelium via western blot and immunohistochemistry. Statistical analysis revealed that attenuated expression of SIRT2 was associated with the International Federation of Gynecology and Obstetrics classification of ovarian cancer. Reduced SIRT2 expression during tumorigenesis failed to repress cyclin-dependent kinase 4 expression, which eventually led to accelerated cell proliferation. Furthermore, the wound healing assay and Transwell assay determined that reduced expression of SIRT2 promoted SOC cell migration and invasion. In conclusion, the results of the current study suggest that SIRT2 has a tumor-suppressor function in ovarian cells and it might be a viable target for further SOC treatment.
\end{abstract}

Correspondence to: Dr Hong Sun, Department of Gynecology, Obstetrics and Gynecology Hospital of Fudan University, 419 Fangxie Road, Shanghai 200011, P.R. China

E-mail: hongsun57@hotmail.com

*Contributed equally

Key words: silent information regulator 2 related enzyme 2, serous ovarian carcinoma, ovarian surface epithelium, FIGO staging, cyclin-dependent kinase 4

\section{Introduction}

Ovarian cancer is one of the most common cancers that affects women (1). Ovarian cancer has the third highest incidence of gynecological oncology, with the incidences of only cervical and uterine cancer being higher amongst women (2). Ovarian epithelial cancer is the most common type of cancer affecting the ovaries and the most lethal of all gynecologic malignancies (2).

The silent information regulator 2 related enzyme 2 (SIRT2) is a nicotinamide adenine dinucleotide-dependent class III histone deacetylase (3). SIRT2 possesses histone or protein deacetylase activity and has an important role in the response to certain types of stress and toxicity (4). SIRT2 is localized in the cytoplasm and nucleus, and is highly expressed in the brain and adipose tissues (5). As SIRT2 participates in the regulation of mitosis and oxidative stress, studies have demonstrated that the expression level of SIRT2 is closely associated with a variety of cancers, including glioma, melanoma, gastric and liver cancer (6-8). Additionally, SIRT2 has been reported to have two opposing roles; there is evidence that it functions as an oncogene, and evidence also exists that suggests it functions as a tumor suppressor gene. A number of studies have indicated that SIRT2 is an oncogene as it is highly expressed, and is associated with vascular invasion and short overall survival in hepatocellular carcinoma (HCC) (9), neuroblastoma (10) and pancreatic cancer (11). However, a number of other studies have suggested that SIRT2 functions as a tumor suppressor based on evidence that SIRT2 mRNA and protein levels are lower in glioma (12), gastric cancer (13) and melanoma (14) compared with normal tissues, and forced expression of SIRT2 inhibits cancer cell proliferation and colony formation in non-small cell lung cancer $(15,16)$. SIRT2 deacetylates a variety of substrates, including histone H4K16, tubulin, p53, p65, forkhead box (FOX) O1, FOXO3, cadherin 1 and cell division cycle 20 (17-21). The specific role of SIRT2 in tumorigenesis is controversial and it is thought that SIRT2 function varies depending on the tissue.

Targeted therapy has been performed at remarkable levels of efficiency and safety in clinical practices in numerous types 
of cancer, including breast cancer, lung cancer and colon cancer (22). However, targeted treatment for ovarian cancer remains challenging as there is a lack of understanding about the molecular mechanisms underlying ovarian tumorigenesis. Thus, it is expected that identifying more pathways that control ovarian cancer tumorigenesis may improve ovarian cancer treatment.

\section{Materials and methods}

Tissue samples and cell lines. Normal ovarian surface epithelial (OSE) tissue samples $(n=11)$ and serous ovarian cancer (SOC) tissue samples $(n=13)$ included for the paired study were collected from the Hospital of Obstetrics and Gynecology of Fudan University (Shanghai, China) between March 10th and December 20th, 2014, following ovarian cancer surgeries (patients were aged from 38 to 65). Further SOC tissue samples $(n=131)$ with complete clinical information were obtained from the Pathology Center of Shanghai Jiaotong University (Shanghai, China). Informed consent was obtained from all patients included in the study. Tissue experiments were approved by the Ethical Committee of Fudan University and Shanghai Jiaotong University (Shanghai, China). The HOSEpiC human OSE cell line, and Hey, HO8910, OVCAR-433, CAOV3 and SKOV3 SOC cell lines were purchased from the Cell Bank of Type Culture Collection of Chinese Academy of Sciences (Shanghai, China). Cells were cultured with RPMI-1640 medium (HyClone; Thermo Fisher Scientific, Inc., Waltham, MA, USA) supplemented with $10 \%$ fetal bovine serum (FBS; Gibco; Thermo Fisher Scientific, Inc.) at $37^{\circ} \mathrm{C}$ at $5 \% \mathrm{CO}_{2}$.

Transfection of cell lines. HOSEpiC human OSE and HO8910 human SOC cells were transfected using Lipofectamine 3000 (Thermo Fisher Scientific, Inc.) with SIRT2 small interfering RNA (siRNA) or an overexpression construct (SIRT2-Myc) that generates SIRT2-Myc fusion proteins, respectively, for $24 \mathrm{~h}$. The overexpression construct was derived from the commercially purchased pCMV-C-Myc plasmid (cat. no. D2672; Beyotime Institute of Biotechnology, Haimen, China) by inserting a SIRT2 gene according to the standard protocol previously described (23). The control groups (NC) were either treated with scramble RNA or empty vector (pCMV-C-Myc). The siRNAs synthesized by Shanghai GenePharma Co., Ltd. (Shanghai, China) were as follows: SIRT2 siRNA-1 forward 5'-GCCUCUAUGACAACCUAGATT-3' and reverse 5'-UCU AGGUUGUCAUAGAGGCTT-3'; SIRT2 siRNA-2 forward 5'-GAUCAGCUAUUUCAAGAAATT-3' and reverse 5'-UUU CUUGAAAUAGCUGAUCTT-3'; SIRT2 siRNA-3 forward 5'-GCACCUUCUACACAUCACATT-3' and reverse 5'-UGU GAUGUGUAGAAGGUGCTT-3'; Scramble forward 5'-UUC UCCGAACGUGUCACGUTT-3' and reverse 5'-ACGUGA CACGUUCGGAGAATT-3'.

Immunohistochemistry (IHC). A total of 131 tumor tissue samples were fixed in $4 \%$ neutralized formaldehyde and embedded in paraffin. Tissue sections $(4 \mu \mathrm{m})$ were processed according to standard immunohistochemical procedures that have been described previously (24). The SIRT2 primary antibody was purchased from Sigma-Aldrich (cat. no. HPA011165; dilution: 1:500, Merck Millipore, Darmstadt, Germany) and the horseradish peroxidase-conjugated secondary antibody was purchased from Fuzhou Maixin Biotech. Co., Ltd. (Fuzhou, China; cat. no. KIT-5005; Ready-to-use). The staining intensities were graded by two pathologists as low (negative or weak staining intensity) or high (medium or strong staining intensity). Images were taken with a Leica DM7000 light microscope equipped with Leica Application Suite software (version 4.5.0; Leica Microsystems, Inc., Buffalo Grove, IL, USA).

Western blot. Western blot was performed as previously described (24). Briefly, cell lines and homogenized tissue samples were lysed with ice cold lysis buffer $(\mathrm{pH} 8.0,50 \mathrm{mM}$ Tris-HCl, $150 \mathrm{mM} \mathrm{NaCl}, 1 \%$ Triton-X 100, $100 \mu \mathrm{g} / \mathrm{ml}$ PMSF). Proteins were quantified via Bradford Protein Assay (Coomassie Brilliant Blue G-250; cat. no. 0615; Amresco, Inc., Framingham, MA, USA) and $20 \mu \mathrm{g}$ protein per sample was loaded in each lane, separated by $10 \%$ SDS-PAGE and then transferred to a nitrocellulose membrane. Membranes were then blocked in $10 \%$ nonfat milk in Tris-buffered saline with $1 \%$ Tween-20 (TBST) for $30 \mathrm{~min}$ at room temperature, followed by incubation with specific primary antibodies at room temperature for $2 \mathrm{~h}$ (anti-SIRT2, cat. no. 12650, dilution: 1:1,000, Cell Signaling Technology, Inc., Danvers, MA, USA; anti-CDK4, cat. no. 12790, dilution: 1:1,000, Cell Signaling Technology, Inc.; anti-CDK6, cat. no. 13331; dilution: 1:1,000, Cell Signaling Technology, Inc.; anti-cyclin D1, cat. no. 2978, dilution: 1:1,000, CST; anti-p16, cat. no. sc-28260, dilution: 1:500, Santa Cruz Biotechnology, Inc., Dallas, TX, USA; anti-actin, cat. no. 3700, dilution: 1:2,000, Cell Signaling Technology, Inc.) Membranes were probed with horseradish peroxidase-conjugated secondary antibodies (Anti-mouse IgG, cat. no. 7076, and anti-rabbit IgG: cat. no. 7074; dilution: 1:2,000; Cell Signaling Technology, Inc.) for $1.5 \mathrm{~h}$ at room temperature. The blots were visualized using an enhanced chemiluminescence reagent (Merck Millipore). Each experiment was conducted for three times followed by band intensity analysis using Image Quant TL software v2005 (version no. 1.1.0.1; non-linear USA, Inc., Durham, NC, USA).

Immunofluorescence. HOSEpiC human OSE and human SOC HO8910 cells were seeded at a density of $1 \times 10^{5}$ cells/well onto coverslips in 6-well plates. Cells were fixed with paraformaldehyde (4\%) for $15 \mathrm{~min}$ followed by Triton-X-100 (0.3\%) treatment at room temperature for $10 \mathrm{~min}$. Bovine serum albumin (3\%, cat. no. V900933; Sigma-Aldrich; Merck Millipore) was used to block non-specific binding site at room temperature for $1 \mathrm{~h}$. Cells were then incubated with primary anti-SIRT2 antibody (cat. no. ab51023, dilution: 1:150; Abcam, Cambridge, UK) overnight at $4^{\circ} \mathrm{C}$. After washed with PBS for 3 times, cells were incubated with the secondary antibody Fluorescein isothiocyanate-conjugated goat anti-rabbit IgG (cat. no. P0186, dilution: 1:250; Beyotime Institute of Biotechnology) in the dark for $1 \mathrm{~h}$ at room temperature, followed by $1 \mathrm{mM}$ DAPI treatment for $5 \mathrm{~min}$ to label the nucleus. The samples were mounted for imaging using Leica DM7000 fluorescence microscope equipped with Leica Application Suite software (version 4.5.0; Leica Microsystems, Inc.). 
Reverse transcription-quantitative polymerase chain reaction $(R T-q P C R)$ analysis. Total RNA was extracted with TRIzol (Thermo Fisher Scientific, Inc., Waltham, MA, USA) according to the manufacturer's protocol. Reverse transcription to produce cDNA was performed by using the Toyobo First-Strand cDNA Synthesis kit (Toyobo Co., Ltd., Osaka, Japan) according to the manufacturer's protocol. CDK4 levels were detected by SYBR Green-based qPCR (SYBR Extaq MixTMII kit; cat. no. RR820A; Takara Bio, Inc., Otsu, Japan) using the Applied Biosystems ${ }^{\circledR} 7500$ Fast Real-Time PCR System (Thermo Fisher Scientific, Inc.). The PCR mixture was amplified by 40 repeated cycles: $95^{\circ} \mathrm{C}$ for $15 \mathrm{sec}, 60^{\circ} \mathrm{C}$ for $30 \mathrm{sec}, 74^{\circ} \mathrm{C}$ for $45 \mathrm{sec}$.

Primer sequences were as follows: CDK4, forward 5'-AGT TCGTGAGGTGGCTTTA-3', reverse 5'-GGGTGCCTTGTC CAGATA-3'; $\beta$-actin, forward 5'-CATCCTCACCCTGAA GTACCC-3', reverse 5'-AGCCTGGATAGCAACGTACAT G-3'. CDK4 mRNA expression was normalized to $\beta$-actin. The $\mathrm{Cq}$ value of CDK4 was measured using the $\Delta \Delta \mathrm{Cq}$ method (25). CDK4 mRNA levels in the treated group (SIRT2-Myc) were compared with untreated groups (NC and HO8910). Results were from three independent experiments performed in triplicate.

Cell Counting Kit-8 assay. The experiment was performed according to the manufacturer's protocol (Cell Counting Kit-8; cat. no. CK04; Dojindo Molecular Technologies, Inc., Kumamoto, Japan). Briefly, HOSEpiC and HO8910 cells pretreated with either SIRT2 siRNA-2 or SIRT2-Myc overexpression construct were seeded on 96-well plates at a density of $5 \times 10^{4}$ cells/well. Cell viabilities were measured at $0,12,24$, $36,48,60$, and $72 \mathrm{~h}$ after transfection by microplate reader at $450 \mathrm{~nm}$. The percentage of relative proliferation ratio was calculated as follows: Relative cell proliferation=number of cells in treatment wells at indicated time/number of cells in corresponding treatment at $0 \mathrm{~h}$.

Transwell assay. HOSEpiC and HO8910 cells pretreated with either SIRT2 siRNA-2 or the SIRT2-Myc overexpression construct were seeded into the upper chamber at a density of $1 \times 10^{5}$ cells/well. $600 \mu 1$ of RPMI-1640 (HyClone; Thermo Fisher Scientific, Inc.) supplemented with $10 \%$ fetal bovine serum (FBS; Gibco; Thermo Fisher Scientific, Inc.) were added into the lower chamber. Cells in the upper chamber were removed following $36 \mathrm{~h}$ incubation at $37^{\circ} \mathrm{C}$, and those that penetrated the membrane to the underside were fixed with methanol for $30 \mathrm{~min}$ at $4^{\circ} \mathrm{C}$, followed by staining with $0.1 \%$ crystal violet for $20 \mathrm{~min}$. Cell numbers in five separate fields at x200 magnification under light microscopy were counted and then averaged.

Wound-healing assay. HOSEpiC and HO8910 cells transfected with SIRT2 siRNA or overexpression construct (SIRT2-Myc), respectively, were seeded on 6-well plates to confluent monolayer in RPMI-1640 supplemented with 10\% FBS. Cells were serum-starved prior to and following scratches to minimize the influence of cell proliferation. Cells were manually scratched using a $20 \mu \mathrm{l}$ sterile pipet tip. The same fields of the wound region were photographed at 0 and $48 \mathrm{~h}$. Wound closure at indicated time points was measured using ImageJ software
(Java 1.6.0_20; National Institutes of Health, Bethesda, MD, USA). Migration rate at $48 \mathrm{~h}$ was expressed as the percentage of the original wound area \pm standard deviation (SD).

Colony formation assay. HOSEpiC and HO8910 cells were transfected with SIRT2 siRNA or overexpression constructs overnight and were then seeded into 12 -well plates at 300 cells per well. Cells were continuously cultured at $37^{\circ} \mathrm{C}$ under $5 \%$ $\mathrm{CO}_{2}$ with $10 \%$ FBS for 7 days. The plates were stained with $200 \mu \mathrm{l}$ crystal violet $(0.1 \%)$ and individual colonies (>50 cells per colony) were counted.

Flow cytometry analysis for cell cycle. HO8910 cells were seeded in 6 -well plates at the density of $2 \times 10^{5}$ cells/well and then transfected with SIRT2 overexpression constructs for 24 h. For cell cycle analysis, cells were harvested and washed twice with cold PBS and then fixed in $70 \%$ ethanol at $-20^{\circ} \mathrm{C}$ for $24 \mathrm{~h}$. The samples were dissolved and washed with PBS 3 times, centrifuged for $5 \mathrm{~min}$ at $420 \mathrm{xg}$ at $4^{\circ} \mathrm{C}$ and then resuspended in PBS with $50 \mu \mathrm{g} / \mathrm{ml}$ RNAse A and incubated at $37^{\circ} \mathrm{C}$ for $30 \mathrm{~min}$. The cell cycle phase was determined by staining with propidium iodide (PI) staining solution $(20 \mu \mathrm{g} / \mathrm{ml})$. Flow cytometry analyses were performed using a FACSCalibur Flow Cytometer and CellQuest software (version 5.1; BD Biosciences, Franklin Lakes, NJ, USA). A total of 10,000 events were collected for final analysis.

Statistical analysis. The $\chi^{2}$ test was used to calculate the significance of differences among three or more groups and paired Student's $t$-test was used to determine the significance of differences between two groups. $\mathrm{P}<0.05$ was considered to indicate a statistically significant difference. All statistical analyses were performed using SPSS software (version no. 13.0; SPSS Inc., Chicago, IL, USA).

\section{Results}

SIRT2 expression level is downregulated in SOC. Expression levels of SIRT2 in 13 cases of SOC and 11 cases of OSE tissue samples were evaluated by IHC. In the OSE cases, 10 out of 11 exhibited high SIRT2 expression, compared with 4 out of 13 cases of SOC that exhibited high SIRT 2 expression (Fig. 1A). Furthermore, SIRT2 was observed predominantly in the nucleus in OSE, while in SOC, no apparent SIRT2 staining was observed in the nucleus (Fig. 1B). Western blot analysis was performed on tissues to further evaluate the expression of SIRT2 in the above tissue samples. Consistent with the IHC results, western blot demonstrated that SIRT2 protein levels in 9 cases of SOC samples were visibly lower than those in 3 cases of OSE samples (Fig. 1C). In addition, SIRT2 expression levels in ovarian cell lines were evaluated by western blot. Compared with the normal human OSE cell line HOSEpiC, all five human SOC cell lines exhibited reduced expression of SIRT2, however, the SKOV3 SOC cell line appeared to have less of a reduction compared with the other four (Fig. 1D). The immunofluorescence assay demonstrated that SIRT2 was localized in the nucleus of HOSEpiC cells, while in HO8910 cells, SIRT2 was predominantly localized in the cytoplasm (Fig. 1E), which confirmed the IHC results. 


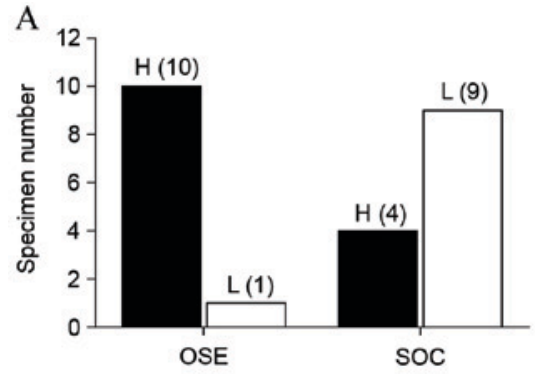

C

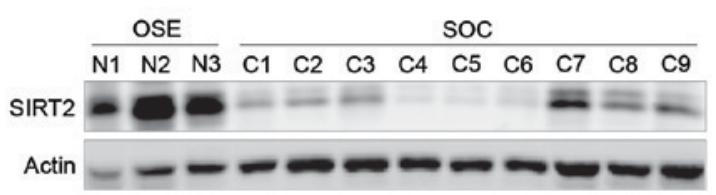

B

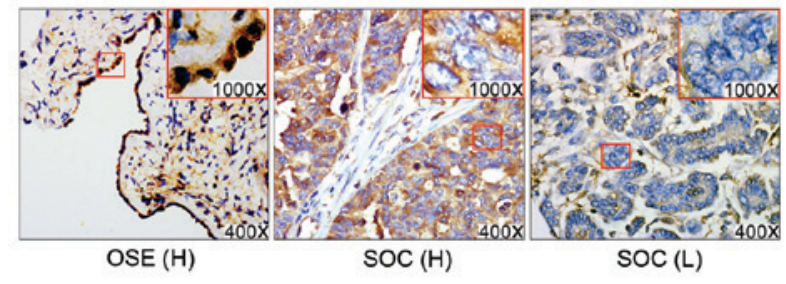

D

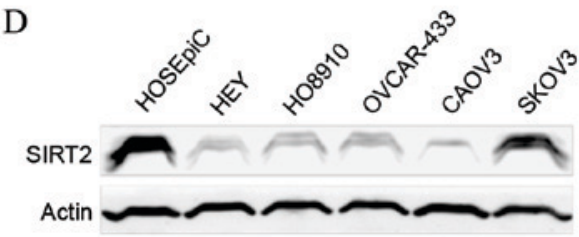

E

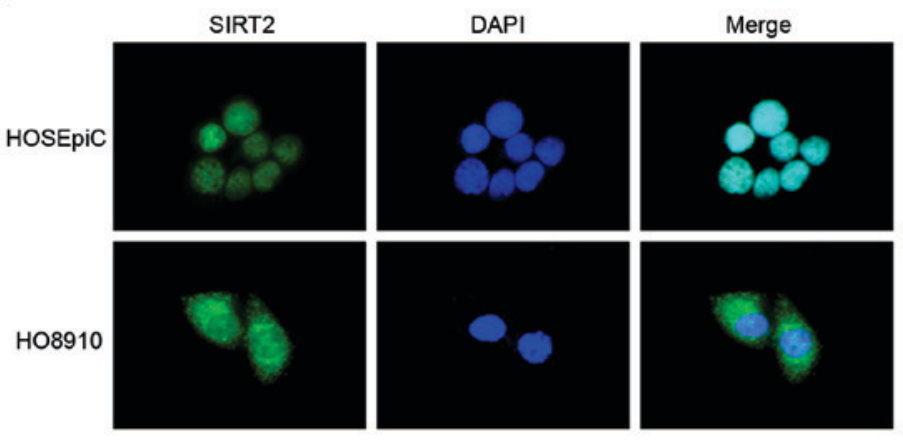

Figure 1. Expression of SIRT2 in OSE and SOC tissues. (A) 10/11 OSE tissue samples, compared with 4/13 SOC tissue samples, exhibited high expression of SIRT2. (B) Images present high expression of SIRT2 in OSE and SOC tissue, and low expression of SIRT2 in SOC, obtained by immunohistochemistry. (C) SIRT2 expression in 3 cases of OSE and 9 cases of SOC, obtained by western blot. (D) SIRT2 expression in normal human OSE cell line HOSEpiC and 5 human SOC cell lines. (E) Immunofluorescence results highlight distinct subcellular localization of SIRT2 in HOSEpiC and HO8910 cells. H, high; L, low; OSE, ovarian surface epithelial; SOC, serous ovarian carcinoma; SIRT2, silent information regulator 2 related enzyme 2.

Reduced expression of SIRT2 is associated with later International Federation of Gynecology and Obstetrics (FIGO) staging of SOC. As there were discrepancies in the expression of SIRT2 in SOC tissue samples (3/9 had reduced SIRT2 expression) and cell lines (4/6 had reduced SIRT2 expression), it is of interest to understand the underlying factors that were associated with SIRT2 levels. Analysis of clinicopathological characteristics and SIRT2 expression (high/low) in 131 SOC samples identified that SIRT2 expression was significantly $(\mathrm{P}<0.017)$ associated with FIGO staging (Table I). There was a higher expression of SIRT2 in early stages (I-II) but lower expression during later stages (III-IV), suggesting a malignant role for SIRT2 during ovarian tumorigenesis. In addition, low expression of SIRT2 was more likely to be associated with lymph node metastasis compared with high expression, although no significant association was observed between lymph node metastasis and SIRT2 levels.

SIRT2 specifically suppresses CDK4 expression in SOC cells. Opposing protein expression patterns of SIRT2 and CDK4 were observed in HOSEpiC normal ovarian cell line compared with HEY, OVCAR-433 and HO8910 SOC cell lines $(\mathrm{P}<0.01$; Fig. 2A), thus, suggesting that SIRT2 may be an upstream regulator of CDK4 in ovarian cell lines. In order to verify this, knockdown of SIRT2 in HOSEpiC cells using SIRT2 siRNAs was performed. CDK4 expression was elevated following transfection with three different SIRT2 siRNAs compared with the control (scramble) siRNA $(\mathrm{P}<0.01$; Fig. 2B). By contrast, overexpression of SIRT2 in HO8910 cells significantly reduced CDK4 protein levels $(\mathrm{P}<0.01$; Fig. 2C). SIRT2 affects target protein levels through transcriptional regulation and post-translational modification (deacetylation) $(26,27)$. Evidence from qPCR results excluded an effect of SIRT2 on CDK4 mRNA transcription as no statistically significant difference was observed between the SIRT2 overexpressing and NC group, indicating that the effect of SIRT2 on CDK4 levels may be predominantly due to post-translational modification of CDK4 by SIRT2 (Fig. 2D). Additionally, the present study also revealed that SIRT2 specifically inhibits CDK4, which regulates the cell cycle through interaction with other members of the cycle, cyclin D1 and tumor suppressor p16. SIRT2 overexpression had no effect on the protein levels of CDK6, cyclin D1 and p16 (Fig. 2E). Flow cytometry determined that overexpression of SIRT2 significantly blocked cell cycle at the G1 phase $(\mathrm{P}<0.05$; Fig. 2F).

Knockdown of SIRT2 promotes ovarian cell proliferation. Consistent with the fact that upregulation of CDK4 induced uncontrolled cell growth (28), knockdown of SIRT2 significantly promoted HOSEpiC cell proliferation compared 
Table I. SIRT2 expression is associated with FIGO staging.

\begin{tabular}{|c|c|c|c|c|}
\hline Variables & $\begin{array}{l}\text { No. of } \\
\text { patients }\end{array}$ & $\begin{array}{c}\text { Low SIRT2 } \\
\text { expression }(n, \%)\end{array}$ & $\begin{array}{c}\text { High SIRT2 } \\
\text { expression }(n, \%)\end{array}$ & P-value \\
\hline \multicolumn{5}{|c|}{ Age (years) } \\
\hline$<50$ & 46 & $20(43.5)$ & $26(56.5)$ & \multirow[t]{2}{*}{0.365} \\
\hline$\geq 50$ & 85 & $44(51.8)$ & $41(48.2)$ & \\
\hline \multicolumn{5}{|c|}{ FIGO stage } \\
\hline I-II & 107 & $47(43.9)$ & $60(56.1)$ & \multirow[t]{2}{*}{0.017} \\
\hline III-IV & 24 & $17(70.8)$ & $7(29.2)$ & \\
\hline \multicolumn{5}{|c|}{ Histological grade } \\
\hline G1-G2 & 89 & $40(44.9)$ & $49(55.1)$ & \multirow[t]{2}{*}{0.192} \\
\hline G3 & 42 & $24(57.1)$ & $18(42.9)$ & \\
\hline \multicolumn{5}{|c|}{ Lymph node metastasis } \\
\hline Yes & 17 & $11(64.7)$ & $6(35.3)$ & \multirow[t]{2}{*}{0.161} \\
\hline No & 114 & $53(46.5)$ & $61(53.5)$ & \\
\hline
\end{tabular}

Data from 131 serous ovarian carcinoma samples. SIRT2, silent information regulator 2 related enzyme 2; FIGO, International Federation of Gynecology and Obstetrics.

with non-transfected HOSEpiC cells, and overexpression of SIRT2 inhibited HO8910 cell proliferation from $48 \mathrm{~h}$ after transfection $(\mathrm{P}<0.05$; Fig. $3 \mathrm{~A}$ and $\mathrm{B})$. Additionally, SIRT2 knockdown significantly increased colony formation in HOSEpiC cells compared with non-transfected HOSEpiC cells and overexpression of SIRT2 decreased the number of colonies in HO8910 samples compared with non-transfected HO8910 cells ( $\mathrm{P}<0.05$; Fig. 3C and D).

Knockdown of SIRT2 increases cell migration and invasion. Knockdown of SIRT2 significantly promoted HOSEpiC cell migration and invasion compared with the non-transfection and scramble group $48 \mathrm{~h}$ post-transfection $(\mathrm{P}<0.05)$ while SIRT2 overexpression inhibited HO8910 cell migration and invasion at $48 \mathrm{~h}$ compared to the non-transfection and $\mathrm{NC}$ group $(\mathrm{P}<0.05$; Fig. 4A and $\mathrm{B})$, suggesting that SIRT2 has an important function in the suppression of ovarian cancer metastasis.

\section{Discussion}

Ovarian epithelial cancer is the most common type of ovarian cancer, accounting for $\sim 90 \%$ of ovarian cancer cases. It has been reported that $\sim 2 / 3$ of ovarian epithelial cancer cases are SOC (29). The FIGO classification system is widely used to evaluate the extent of ovarian cancer and is the basis for post-surgical treatment (30). As the early stages of ovarian cancer are asymptomatic, most ovarian cancers are diagnosed in the advanced FIGO stages (31), which means that treatment options for patients are limited and this accounts for the relatively low five-year survival rate and high recurrence rate worldwide (32). Investigation of the molecular pathogenesis of ovarian cancer is required to develop novel therapies and improve patient outcomes.

SIRT2 is a deacetylase and it has been reported that it is functionally associated with tumorigenesis, either as a tumor suppressor or an oncogene $(3,6)$. It has been previously reported that SIRT2 expression is low in glioma, melanoma and gastric cancer and that high levels are present in acute myeloid leukemia, neuroblastoma, pancreatic cancer and HCC (6). Thus, the specific function of SIRT2 appears to depend on the features of specific tumors. Although SIRT2 has been extensively investigated in various types of cancer (6-16), SIRT2 expression in ovarian cancer has not yet been investigated. In the current study, reduced expression of SIRT2 in SOC compared with normal OSE was confirmed by IHC and western blot. Furthermore, low expression of SIRT2 was significantly associated with late FIGO stage SOC, suggesting that SIRT2 is important in the prevention of ovarian cancer metastasis. Statistical analysis did not indicate that low expression of SIRT2 was associated with increased lymph node metastasis, as peritoneal dissemination and local direct spread are the two main methods of ovarian cancer metastasis. The accuracy of FIGO staging is an important factor if ovarian cancer patients are to be treated effectively, creating a high demand for gynecological oncologists to comprehensively consider patients' surgical and pathological indications (33). Omission of insidious metastasis does occur in a small portion of patients resulting in inadequate treatment (as it is difficult to examine insidious metastasis, the seriousness of disease may be underrated) and recurrence. Thus, it is imperative to apply other methods to complement the FIGO system. The present study demonstrated that SIRT2 levels are significantly associated with FIGO staging of SOC. In early FIGO stages (I-II) there are higher levels of SIRT2, while in late FIGO stages III-IV) there are reduced levels of SIRT2 expression, indicating that SIRT2 expression levels may be a valuable reference for FIGO evaluation.

SIRT2 is reported to be downstream of several CDKs and may be phosphorylated at S331 or S368 by certain CDKs, including CDK1, CDK2, and CDK5, which are essential for the catalytic activity of SIRT2 (34). The present study 

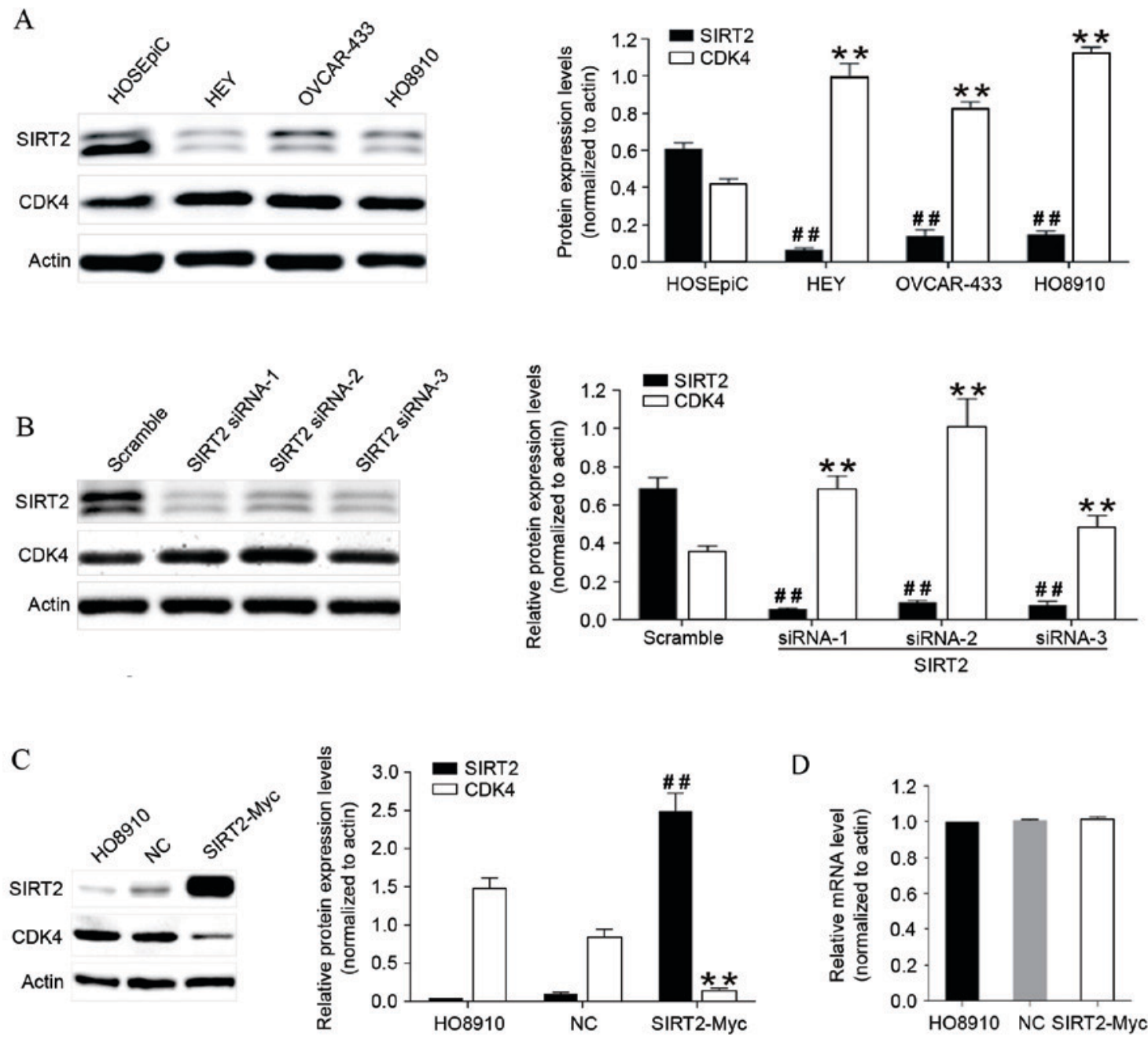

$\mathrm{E}$
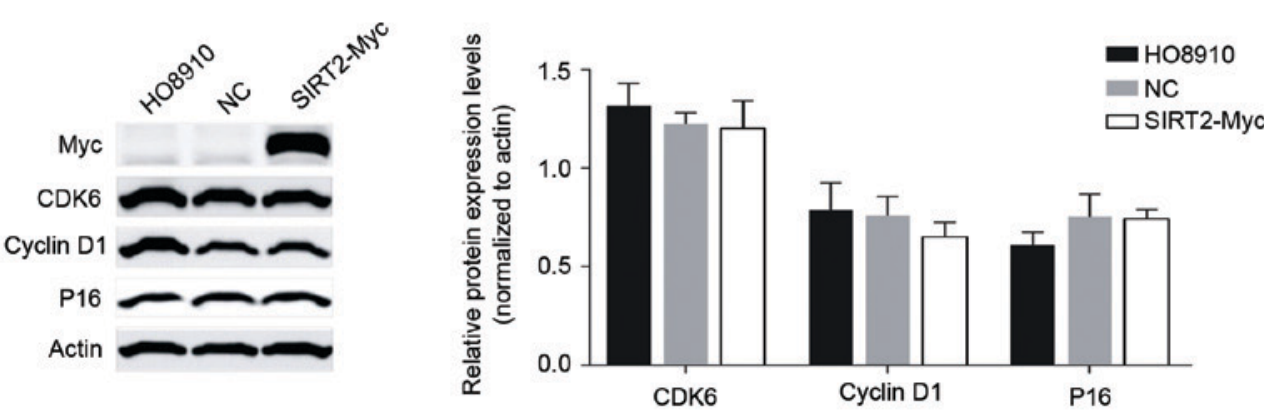

$\mathrm{F}$

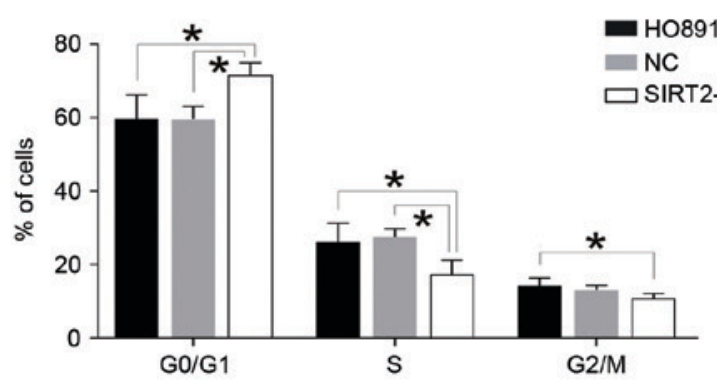

Figure 2. SIRT2 is an upstream regulator of CDK4. (A) Protein expression of CDK4 and SIRT2 in serous ovarian carcinoma cell lines ( ${ }^{* *} \mathrm{P}<0.01$ vs. CDK4 in HOSEpiC; ${ }^{\# \#} \mathrm{P}<0.01$ vs. SIRT2 in HOSEpiC). (B) Endogenous SIRT2 expression in HOSEpiC cells was reduced by SIRT2 siRNA 1-3 (" $\mathrm{P}<0.01$ vs. CDK4 in scramble group; ${ }^{\# \#} \mathrm{P}<0.01$ vs. SIRT2 in scramble group). (C) Overexpression of SIRT2-Myc in HO8910 cells significantly elevated the SIRT2 protein level $\left({ }^{\# \#} \mathrm{P}<0.01\right.$ vs. SIRT2 in HO8910 and NC) and repressed CDK4 expression ( ${ }^{* *} \mathrm{P}<0.01$ vs. CDK4 in HO8910 and NC). (D) Overexpression of SIRT2 did not reduce CDK4 mRNA levels. (E) SIRT2 overexpression did not act on the expression of other CDK4-associated proteins. (F) Overexpression of SIRT2 significantly increased cell cycle arrest at the G1 phase ("P $<0.05$, comparison indicated by brackets). SIRT2, silent information regulator 2 related enzyme 2; CDK, cyclin-dependent kinase; siRNA, small interfering RNA; NC, negative control. 


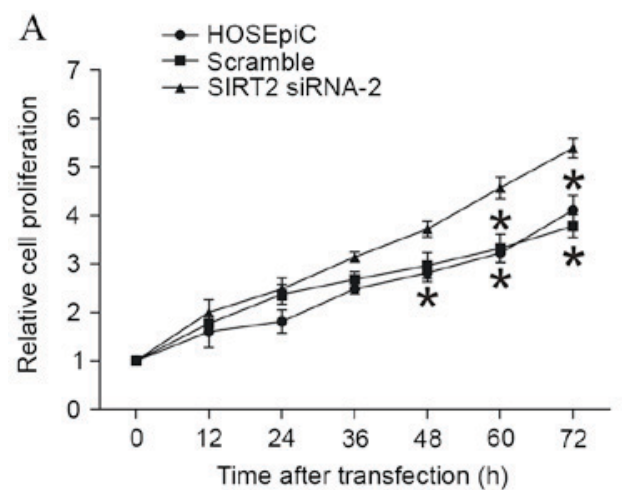

$\mathrm{C}$

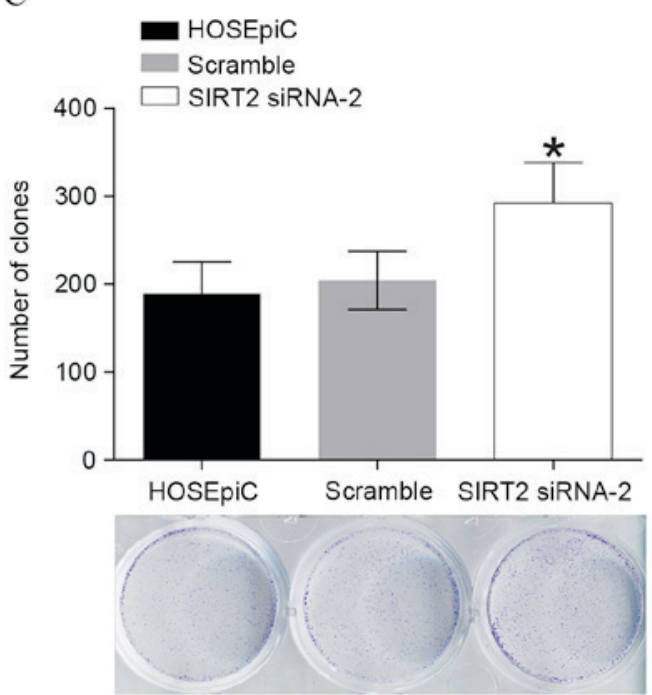

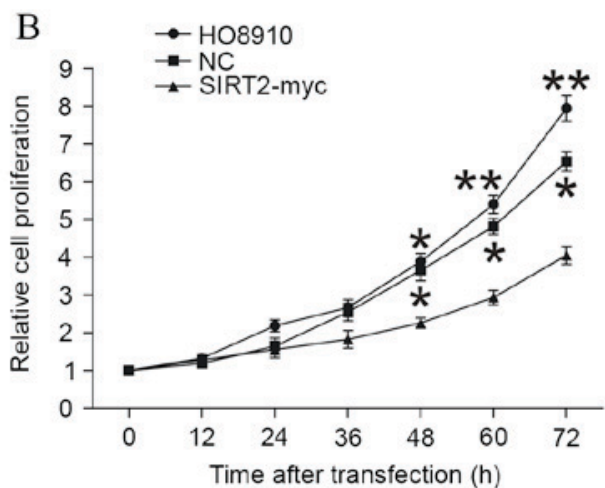

D

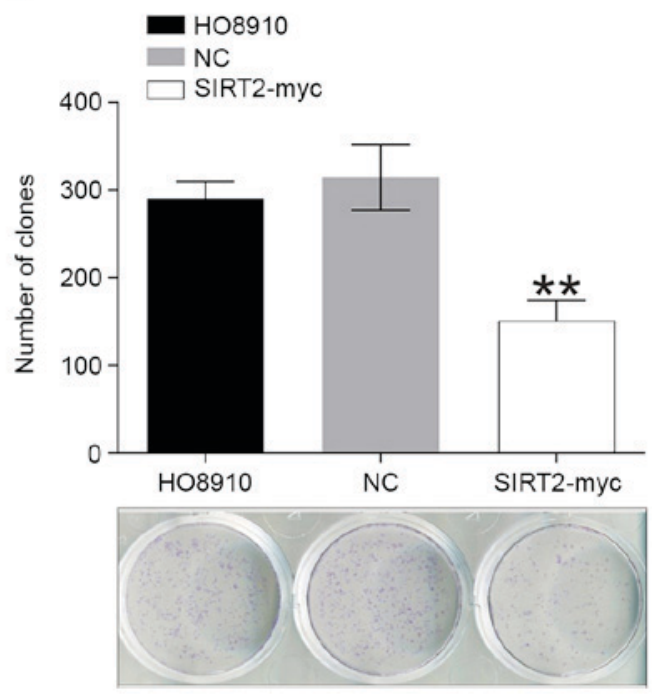

Figure 3. Effect of SIRT2 on cell proliferation. (A) SIRT2 knockdown in HOSEpiC cells enhanced cell proliferation ("P<0.05 vs. HOSEpiCSIRT2 siRNA-2). (B) Overexpression of SIRT2 in HO8910 cells suppressed cell proliferation (" $\mathrm{P}<0.05$ and ${ }^{* *} \mathrm{P}<0.01$ vs. SIRT2-myc). (C) SIRT2 knockdown in HOSEpiC cells increased cell colony formation ("P $<0.05$ vs. HOSEpiC and Scramble). (D) Overexpression of SIRT2 in HO8910 cells inhibited cell colony formation ("* $\mathrm{P}<0.01$ vs. HO8910 and NC). SIRT2, silent information regulator 2 related enzyme 2; NC, negative control; siRNA, small interfering RNA.

provided evidence for a novel SIRT2 regulatory pathway where SIRT2 specifically represses CDK4 expression. Therefore, SIRT2 may affect CDK4 expression through interacting with the CDK4 promoter (transcriptional regulation) or by deacetylating CDK4 (post-translational regulation). The current study excluded the former scenario by measuring CDK4 mRNA levels following SIRT2 overexpression, this suggested that SIRT2 regulation of CDK4 expression may be predominantly mediated via deacetylation of CDK4. Reduced expression of SIRT2 in OSE may cause an abnormal increase in CDK4, which is an established carcinogen.

Consistent with the findings of the present study, Sui et al (35). Observed upregulation of CDK4 expression and nuclear accumulation of CDK4 in malignant ovarian cancers, which may explain the CDK4-induced cell proliferation. In addition to ovarian cancer, elevated expression of CDK4 has also been observed in other malignancies. CDK-targeted therapies, including CDK4 inhibitors, have attracted a lot of attention as they have promising therapeutic effects in breast, lung and colon cancer (28). Wang et al (36) demonstrated that CDK4 is degraded via the ubiquitin pathway. As SIRT2 deacetylates multiple lysine-acetylated proteins located in the cytoplasm and nucleus, and CDK4 has multiple lysine sites that may be exposed to acetyl-transferases, nuclear CDK4 may initially be deacetylated by SIRT2 and then transported to the cytoplasm where it is subject to ubiquitin-dependent degradation. CDK4 and CDK6 are protein kinases that bind cyclin D1 or its competitive inhibitor, p16, to regulate cell-cycle progression (37). Although CDK4 and CDK6 have similar biological functions, the present study demonstrated that SIRT2 specifically acted on CDK4 rather than other proteins, including CDK6 and p16. This combined evidence suggests that SIRT2 acts as a tumor suppressor.

In vitro experiments further supported an anti-cancer function for SIRT2. Overexpression of SIRT2 arrested the ovarian cancer cell line HO8910 cells at the G1 phase. Knockdown of SIRT2 enhanced the proliferation rate of normal ovarian cells, while overexpression of SIRT2 significantly reduced the cell proliferation rate. Furthermore, overexpression of SIRT2 reduced cell clone formation, migration and invasion, whereas knockdown of SIRT2 led to the opposite effects. In conclusion, the current study reported a reduction in SIRT2 expression in ovarian malignancy and identified a novel pathway involving SIRT2/CDK4. These findings are expected to improve the understanding of the tumorigenesis of ovarian cancer. 
A
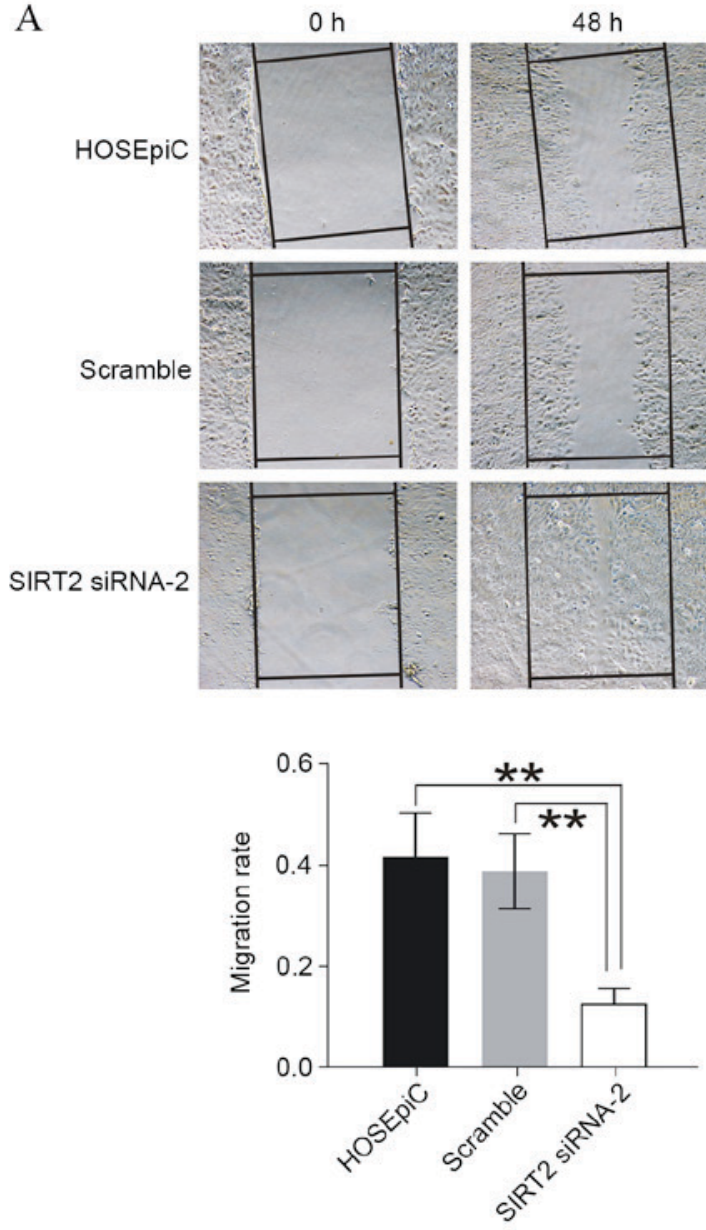

$48 \mathrm{~h}$
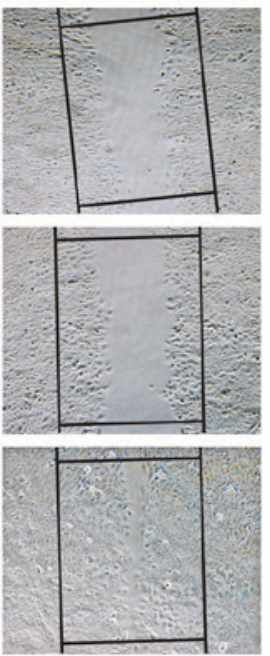

B
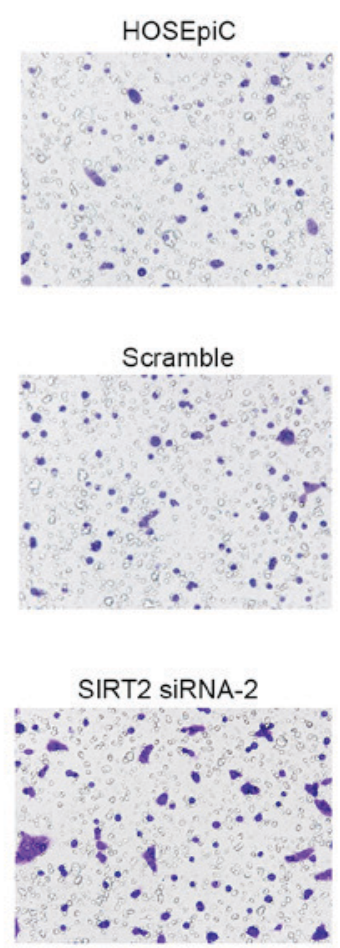

HO8910
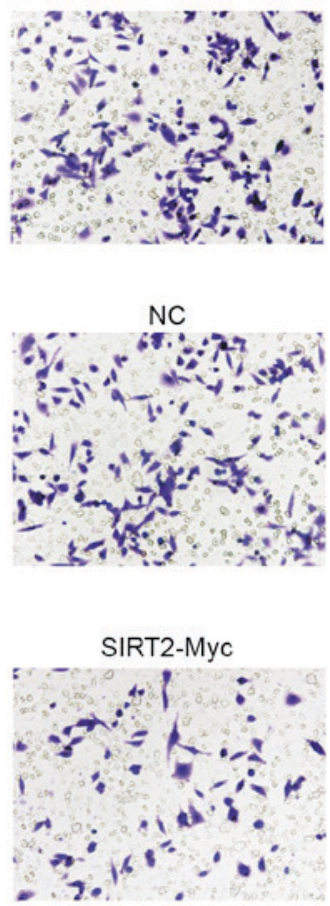

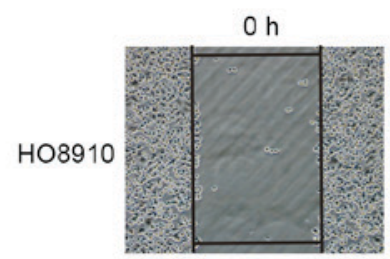

$48 \mathrm{~h}$

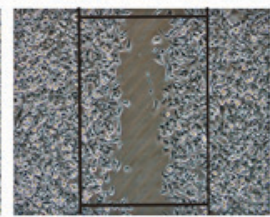

NC

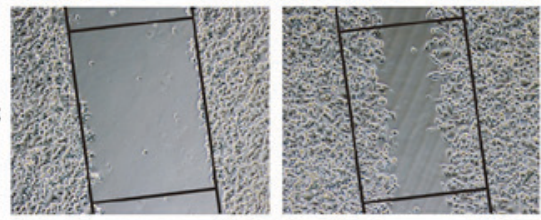

SIRT2-Myc
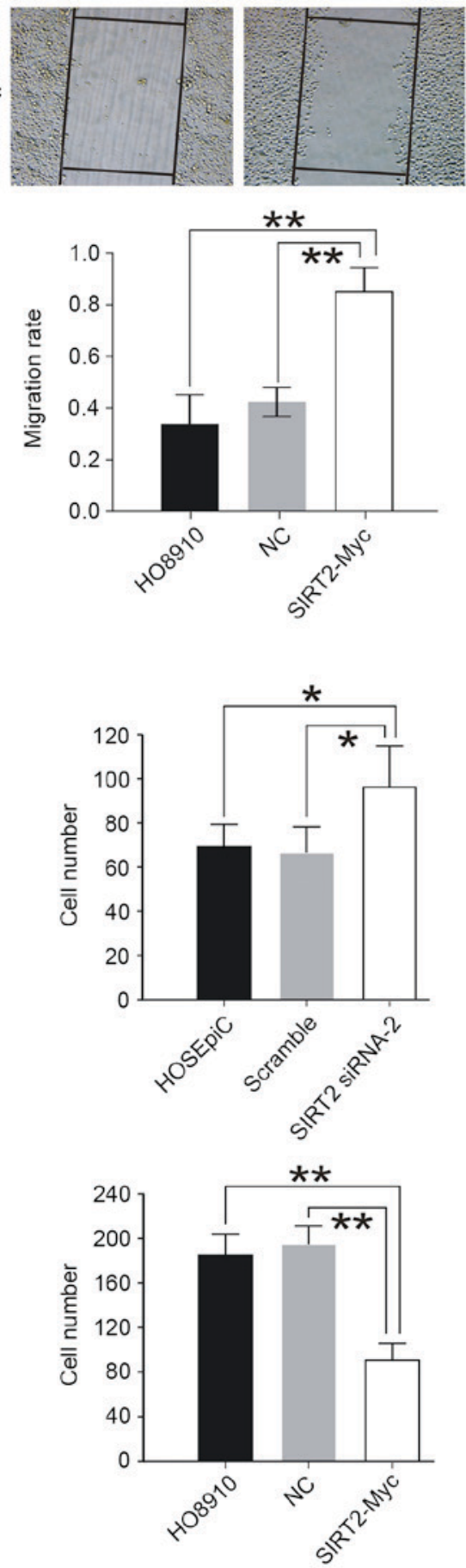

Figure 4. Effect of SIRT2 on ovarian cell migration and invasion ability. (A) Images demonstrate that SIRT2 knockdown in HOSEpiC cells promoted cell migration, while overexpression of SIRT2 in HO8910 cells reduced cell migration. Migration rate at $48 \mathrm{~h}$ was expressed as the percentage of the original wound area. (B) Images of Transwell invasion assay in HOSEpiC and HO8910 cells following knockdown or overexpression of SIRT2, respectively. SIRT2 repression induced cell invasion, while overexpression inhibited cell invasion $\left({ }^{*} \mathrm{P}<0.05,{ }^{* *} \mathrm{P}<0.01\right.$, comparison indicated by brackets). SIRT2, silent information regulator 2 related enzyme 2; siRNA, small interfering RNA; NC, negative control. 


\section{Acknowledgements}

The present study was funded by Shanghai Science and Technology Committee (grant no. 14ZR1404100).

\section{References}

1. Vargas-HernándezVM,Moreno-EutimioMA,Acosta-AltamiranoG and Vargas-Aguilar VM: Management of recurrent epithelial ovarian cancer. Gland Surg 3: 198-202, 2014.

2. Jemal A, Bray F, Center MM, Ferlay J, Ward E and Forman D: Global cancer statistics. CA Cancer J Clin 61: 69-90, 2011.

3. Imai S, Armstrong CM, Kaeberlein M and Guarente L: Transcriptional silencing and longevity protein Sir2 is an NAD-dependent histone deacetylase. Nature 403: 795-800, 2000.

4. Dan L, Klimenkova O, Klimiankou M, Klusman JH, van den Heuvel-Eibrink MM, Reinhardt D, Welte K and Skokowa J: The role of sirtuin 2 activation by nicotinamide phosphoribosyltransferase in the aberrant proliferation and survival of myeloid leukemia cells. Haematologica 97: 551-559, 2012.

5. Wilson JM, Le VQ, Zimmerman C, Marmorstein R and Pillus L: Nuclear export modulates the cytoplasmic Sir2 homologue Hst2. EMBO Rep 7: 1247-1251, 2006.

6. Cha YI and Kim HS: Emerging role of sirtuins on tumorigenesis: Possible link between aging and cancer. BMB Rep 46: 429-438, 2013.

7. Li Y, Dai D, Lu Q, Fei M, Li M and Wu X: Sirt2 suppresses glioma cell growth through targeting NF- $\kappa \mathrm{B}-\mathrm{miR}-21$ axis. Biochem Biophys Res Commun 441: 661-667, 2013.

8. Karwaciak I, Gorzkiewicz M, Ryba K, Dastych J, Pulaski L and Ratajewski M: AC-93253 triggers the downregulation of melanoma progression markers and the inhibition of melanoma cell proliferation. Chem Biol Interact 236: 9-18, 2015.

9. Chen J, Chan AW, To KF, Chen W, Zhang Z, Ren J, Song C, Cheung YS, Lai PB, Cheng SH, et al: SIRT2 overexpression in hepatocellular carcinoma mediates epithelial to mesenchymal transition by protein kinase $\mathrm{B} / \mathrm{glycogen}$ synthase kinase- $3 \beta$ / $\beta$-catenin signaling. Hepatology 57: 2287-2298, 2013.

10. Pizarro JG, Folch J, Esparza JL, Jordan J, Pallàs M and Camins A: A molecular study of pathways involved in the inhibition of cell proliferation in neuroblastoma B65 cells by the GSK-3 inhibitors lithium and SB-415286. J Cell Mol Med 13: 3906-3917, 2009.

11. Zhao D, Zou SW, Liu Y, Zhou X, Mo Y, Wang P, Xu YH, Dong B, Xiong Y, Lei QY and Guan KL: Lysine-5 acetylation negatively regulates lactate dehydrogenase $\mathrm{A}$ and is decreased in pancreatic cancer. Cancer Cell 23: 464-476, 2013.

12. Hiratsuka M, Inoue T, Toda T, Kimura N, Shirayoshi Y, Kamitani H, Watanabe T, Ohama E, Tahimic CG, Kurimasa A and Oshimura M: Proteomics-based identification of differentially expressed genes in human gliomas: Down-regulation of SIRT2 gene. Biochem Biophys Res Commun 309: 558-566, 2003

13. Peters CJ, Rees JR, Hardwick RH, Hardwick JS, Vowler SL, Ong CA, Zhang C, Save V, O'Donovan M, Rassl D, et al: A 4-gene signature predicts survival of patients with resected adenocarcinoma of the esophagus, junction and gastric cardia. Gastroenterology 139: 1995-2004.e15, 2010.

14. Lennerz V, Fatho M, Gentilini C, Frye RA, Lifke A, Ferel D, Wölfel C, Huber C and Wölfel T: The response of autologous T cells to a human melanoma is dominated by mutated neoantigens. Proc Natl Acad Sci USA 102: 16013-16018, 2005.

15. Li Z, Xie QR, Chen Z, Lu S and Xia W: Regulation of SIRT2 levels for human non-small cell lung cancer therapy. Lung Cancer 82: 9-15, 2013

16. Xu W, Jiang K, Shen M, Qian Y and Peng Y: SIRT2 suppresses non-small cell lung cancer growth by targeting JMJD2A. Biol Chem 396: 929-936, 2015.

17. Mangas-Sanjuan V, Oláh J, Gonzalez-Alvarez I, Lehotzky A, Tőkési N, Bermejo M and Ovádi J: Tubulin acetylation promoting potency and absorption efficacy of deacetylase inhibitors. Br J Pharmacol 172: 829-840, 2015.
18. Wang F, Chan $\mathrm{CH}$, Chen $\mathrm{K}$, Guan X, Lin HK and Tong Q: Deacetylation of FOXO3 by SIRT1 or SIRT2 leads to Skp2-mediated FOXO3 ubiquitination and degradation. Oncogene 31: 1546-1557, 2012.

19. Hoffmann G, Breitenbücher F, Schuler $M$ and Ehrenhofer-Murray AE: A novel sirtuin 2 (SIRT2) inhibitor with p53-dependent pro-apoptotic activity in non-small cell lung cancer. J Biol Chem 289: 5208-5216, 2014.

20. Kim HS, Vassilopoulos A, Wang RH, Lahusen T, Xiao Z, Xu X, $\mathrm{Li} \mathrm{C}$, Veenstra TD, Li B, Yu H, et al: SIRT2 maintains genome integrity and suppresses tumorigenesis through regulating APC/C activity. Cancer Cell 20: 487-499, 2011.

21. Jiang W, Wang S, Xiao M, Lin Y, Zhou L, Lei Q, Xiong Y, Guan KL and Zhao S: Acetylation regulates gluconeogenesis by promoting PEPCK1 degradation via recruiting the UBR5 ubiquitin ligase. Mol Cell 43: 33-44, 2011.

22. Tobin NP, Foukakis T, De Petris L and Bergh J: The importance of molecular markers for diagnosis and selection of targeted treatments in patients with cancer. J Intern Med 278: 545-570, 2015.

23. Wu J, Zhang YC, Suo WH, Liu XB, Shen WW, Tian H and Fu GH Induction of anion exchanger-1 translation and its opposite roles in the carcinogenesis of gastric cancer cells and differentiation of K562 cells. Oncogene 29: 1987-1996, 2010.

24. Cui Y, Li SB, Peng XC, Wu J and Fu GH: Trastuzumab inhibits growth of HER2-negative gastric cancer cells through gastrin-initialized CCKBR signaling. Dig Dis Sci 60: 3631-3641, 2015.

25. Livak KJ and Schmittgen TD: Analysis of relative gene expression data using real-time quantitative PCR and the 2(-Delta Delta C (T)) Method. Methods 25: 402-408, 2001.

26. Liu PY, Xu N, Malyukova A, Scarlett CJ, Sun YT, Zhang XD, Ling D, Su SP, Nelson C, Chang DK, et al: The histone deacetylase SIRT2 stabilizes Myc oncoproteins. Cell Death Differ 20: 503-514, 2013.

27. Lin R, Tao R, Gao X, Li T, Zhou X, Guan KL, Xiong Y and Lei QY: Acetylation stabilizes ATP-citrate lyase to promote lipid biosynthesis and tumor growth. Mol Cell 51: 506-518, 2013.

28. Dickson MA: Molecular pathways: CDK4 inhibitors for cancer therapy. Clin Cancer Res 20: 3379-3383, 2014.

29. Nezhat FR, Apostol R, Nezhat C and Pejovic T: New insights in the pathophysiology of ovarian cancer and implications for screening and prevention. Am J Obstet Gynecol 213: 262-267, 2015.

30. Zeppernick F and Meinhold-Heerlein I: The new FIGO staging system for ovarian, fallopian tube, and primary peritoneal cancer. Arch Gynecol Obstet 290: 839-842, 2014.

31. Zapardiel I, Diestro MD and Aletti G: Conservative treatment of early stage ovarian cancer: Oncological and fertility outcomes. Eur J Surg Oncol 40: 387-393, 2014.

32. Jackson AL, Eisenhauer EL and Herzog TJ: Emerging therapies: Angiogenesis inhibitors for ovarian cancer. Expert Opin Emerg Drugs 20: 331-346, 2015.

33. Rutten MJ, Boldingh JH, Schuit E, Trum H, van Driel W, Mol BW, Kenter GG and Buist MR: Development and internal validation of a prognostic model for survival after debulking surgery for epithelial ovarian cancer. Gynecol Oncol 135: 13-18, 2014.

34. Pandithage R, Lilischkis R, Harting K, Wolf A, Jedamzik B, Lüscher-Firzlaff J, Vervoorts J, Lasonder E, Kremmer E, Knöll B and Lüscher B: The regulation of SIRT2 function by cyclin-dependent kinases affects cell motility. J Cell Biol 180: 915-929, 2008

35. Sui L, Dong Y, Ohno M, Goto M, Inohara T, Sugimoto K, Tai Y, Hando T and Tokuda M: Inverse expression of Cdk4 and p16 in epithelial ovarian tumors. Gynecol Oncol 79: 230-237, 2000.

36. Wang $\mathrm{H}$, Goode $\mathrm{T}$, Iakova $\mathrm{P}$, Albrecht $\mathrm{JH}$ and Timchenko NA: C/EBPalpha triggers proteasome-dependent degradation of cdk4 during growth arrest. EMBO J 21: 930-941, 2002.

37. Ortega S, Malumbres M and Barbacid M: Cyclin D-dependent kinases, INK4 inhibitors and cancer. Biochim Biophys Acta 1602: 73-87, 2002. 